\title{
Communication \\ Plasticity of Proinflammatory Macrophages Depends on Their Polarization Stage during Human MSC Immunomodulation-An In Vitro Study Using THP-1 and Human Primary Macrophages
}

\author{
Constantin Ißleib ${ }^{1,2, * \mathbb{D}}$, Susanne $\operatorname{Kurz}^{1,2}$, Samuel Scholl ${ }^{1,2}$, Bettina Amberg ${ }^{1,2}$ and Juliane Spohn ${ }^{1,2} \mathbb{D}$ \\ 1 Fraunhofer Institute for Ceramic Technologies and Systems IKTS, Perlickstr. 1, 04103 Leipzig, Germany; \\ susanne.kurz@ikts.fraunhofer.de (S.K.); samuel.scholl@ikts.fraunhofer.de (S.S.); \\ bettina.amberg@gmx.de (B.A.); juliane.spohn@ikts.fraunhofer.de (J.S.) \\ 2 Fraunhofer Institute for Cell Therapy and Immunology IZI, Perlickstr. 1, 04103 Leipzig, Germany \\ * Correspondence: constantin.issleib@ikts.fraunhofer.de
}

\section{check for} updates

Citation: Ißleib, C.; Kurz, S.; Scholl, S.; Amberg, B.; Spohn, J. Plasticity of Proinflammatory Macrophages Depends on Their Polarization Stage during Human MSC

Immunomodulation-An In Vitro Study Using THP-1 and Human Primary Macrophages. Immuno 2021, 1,518-528. https://doi.org/10.3390/ immuno1040036

Academic Editor: Mary Poupot

Received: 13 October 2021

Accepted: 19 November 2021

Published: 24 November 2021

Publisher's Note: MDPI stays neutral with regard to jurisdictional claims in published maps and institutional affiliations.

Copyright: (c) 2021 by the authors. Licensee MDPI, Basel, Switzerland. This article is an open access article distributed under the terms and conditions of the Creative Commons Attribution (CC BY) license (https:// creativecommons.org/licenses/by/ $4.0 /)$.

\begin{abstract}
Human mesenchymal stem cells (hMSCs) are well-known for their immunomodulatory potential. In recent clinical trials and in vivo studies, hMSCs were used as therapeutic measures to dampen inflammation. In this context, their effect on macrophages in vivo has been described to induce a phenotype change shifting from a proinflammatory to an anti-inflammatory environment. Despite several in vitro studies that investigated the potential of hMSCs to inhibit the polarization of macrophages into the proinflammatory M1 subtype, it is still unclear if hMSCs affect polarized M1 macrophages or if they control the environment by inhibiting the M1 polarization of unpolarized macrophages. Here, a comparative in vitro investigation of hMSC immunomodulation via soluble factors concerning the influence on the polarization of macrophages to M1 and on polarized M1 macrophages is presented. Human primary monocyte-derived macrophages (hMDMs) as well as THP-1 cells were used for this investigation. The macrophage subtype was analyzed by gene expression as well as cytokine secretion. hMSCs affected cytokine secretion of polarizing macrophages, while changes in gene expression were evident in polarized M1 macrophages. These effects were observed in THP-1 and hMDM macrophages. In conclusion, we suggest that hMSCs implement their immunomodulatory effects on polarizing and polarized macrophages in different ways.
\end{abstract}

Keywords: macrophage polarization; macrophage plasticity; MSC immunomodulation; inflammation; in vitro

\section{Introduction}

Macrophages are known for their plasticity referring to their capability to polarize into various subtypes [1]. The differences between these subtypes are well-established and are categorized due to their functions. Generally, there is the proinflammatory M1 subtype, which largely creates the inflammatory environment, and the anti-inflammatory M2 subtype, which dampens inflammation and promotes wound healing. Macrophage populations during the resolution of an inflammation in vivo are often described to change from an M1 to an M2 subtype population, possibly by switching the phenotype [2,3]. However, the change in subtype population is also hypothesized to happen by driving new monocytes towards M2 macrophages [4]. Hence, a closer look at the ability of macrophages to change subtypes can be beneficial to an understanding of inflammation resolution.

Mesenchymal stem cells (MSCs) resolve inflammation due to their immunomodulatory capabilities. In fact, MSC interaction with local cells, such as progenitors and immune cells, is crucial in wound healing. Particularly, their immunomodulatory effects on macrophages in inflammation are of great interest [3,5]. This is emphasized by their frequent use in clinical trials against chronic inflammation [6]. In order to exercise their 
immunomodulatory potential, MSCs recognize inflammatory cues from the microenvironment such as proinflammatory cytokines. However, depending on the stage of wound healing, microenvironment cues may change and push towards proliferative or remodeling signaling pathways [7]. In acute inflammation and direct immunomodulation of macrophages, MSCs have been reported to exercise their immunomodulating mechanisms on macrophages via indoleamine 2,3-dioxygenase (IDO) activity or via soluble factors, such as IL-1ra, IL-10, PGE 2 , and TSG-6, generally upon stimulation [8,9].

Special care must be taken when comparing immunomodulatory functions of MSCs between species and even donors. Fundamental differences in the immunosuppressive actions of MSCs between species have been reported [10]. Other studies reported donor variations of immunomodulatory capacity of human MSCs from the same source [11-13].

In the context of MSC-driven immunomodulation of macrophages, $\mathrm{PGE}_{2}$ is frequently considered to be the main active soluble factor. It was shown that murine MSCs significantly changed the pro- and anti-inflammatory cytokine secretion during M1 polarization of murine primary macrophages via $\mathrm{PGE}_{2}[14,15]$. Manferdini et al. showed that $\mathrm{PGE}_{2}$ created the same anti-inflammatory effect on human polarized M1 monocyte-derived macrophages (MDMs) as a coculture with human MSCs [16].

In vitro investigations of immunomodulation of inflammatory macrophages benefit from qualifying cell lines as adequate substitutes for primary cells, eliminating donor variability. The polarization of THP-1 macrophages (human monocytic cell line) has been compared in depth with human MDMs, stating that observations in the THP-1 model system should be confirmed whenever it is used in a new experimental setting [17]. Vasandan et al. showed that immunomodulation of polarizing M1 macrophages via human MSCs was comparable when using THP-1 macrophages and human MDMs [18].

In addition to the fact that the experiments in the various publications mostly focus either on immunomodulatory effects of MSCs on polarizing macrophages or on immunomodulatory effects of MSCs on already polarized macrophages, they also differ considerably in the protocols regarding inflammatory polarization of macrophages, making comparison difficult.

Here, we present our results of the direct comparison of the effects of human bone marrow MSCs (hMSCs) on polarizing vs. polarized proinflammatory THP-1 macrophages using the most widely used polarization strategy, which includes the stimulation with LPS and IFN- $\gamma$. The effects of using $\mathrm{PGE}_{2}$ vs. the conditioned medium of hMSCs that were primed with M1-conditioned media (M1-primed), as well as using the conditioned medium of M1-primed hMSCs vs. direct cell contact, were investigated. The transferability of the results to human MDMs was additionally confirmed.

\section{Materials and Methods}

\subsection{Isolation of Primary Human Bone Marrow Mesenchymal Stem Cells (hMSCs)}

hMSCs were isolated from bone marrow donations of healthy donors (male, 20-30 years old). All donors gave written consent, and the isolation was approved by an ethics commission (University of Leipzig no. 486/17-ek-001). For the isolation, the bone marrow aspirate was diluted with PBS at a 1:2 ratio. Then, $4 \mathrm{~mL}$ of the bone marrow dilution was diluted with $3.5 \mathrm{~mL}$ Ficoll Paque Premium (GE Healthcare, Chicago, IL, USA), followed by a density gradient centrifugation at $400 \times g$ for $40 \mathrm{~min}$. The white mononuclear cell fraction was isolated, washed with PBS once, and centrifuged at $400 \times g$ for $10 \mathrm{~min}$. The resulting cell pellet was resuspended in DMEM $1 \mathrm{~g} / \mathrm{L}$ glucose, glutaMAX, and pyruvate (Thermo Fisher Scientific, Waltham, MA, USA) plus $10 \%$ fetal calf serum (FCS) (Thermo Fisher Scientific, Waltham, MA, USA) and 1\% penicillin/streptomycin (Thermo Fisher Scientific, Waltham, MA, USA), and cells were transferred to cell-culturing flasks. Only passages 2 to 4 were used in this study. hMSCs were characterized according to the ISCT consensus statement (Dominici et al., 2006). Briefly, surface expression was checked to be above $95 \%$ for CD73, CD90, and CD105, as well as an expression below $5 \%$ for CD11 or CD14, CD34, CD45, CD79a or CD19, and HLA-DR protein using the human 
MSC analysis kit (BD Bioscience, San Jose, USA). Additionally, hMSCs were successfully differentiated to adipocytes, chondrocytes, and osteoblasts (Supplementary Materials Figure S1). Briefly, the differentiation medium was replaced every 3-4 days. Adipogenic differentiation was achieved using $1 \mu \mathrm{M}$ dexamethasone, $5 \mu \mathrm{g} / \mathrm{mL}$ human insulin solution, and $50 \mu \mathrm{M}$ indomethacin (all Sigma Aldrich, Steinheim, Germany) in DMEM $4.5 \mathrm{~g} / \mathrm{L}$ glucose, glutaMAX, and pyruvate (Thermo Fisher Scientific, Waltham, MA, USA) on $1 \times 10^{4} \mathrm{hMSCs} / \mathrm{cm}^{2} / 0.5 \mathrm{~mL}$. After 14 days, adipocytes were stained with il Red O (Sigma Aldrich, Steinheim, Germany). Chondrogenic differentiation was achieved using $100 \mathrm{nM}$ dexamethason, $50 \mu \mathrm{g} / \mathrm{mL}$ L-ascorbic acid 2-phosphate sesquimagnesic salt, $40 \mathrm{\mu g} / \mathrm{mL}$ L-proline (all Sigma Aldrich, Steinheim, Germany), $10 \mathrm{ng} / \mathrm{mL}$ human recombinant TGF-beta 3 (PeproTech, Hamburg, Germany), and $500 \mathrm{ng} / \mathrm{mL}$ human recombinant BMP-2/BMP-7 heterodimer protein (R\&D Systems, Inc., Minneapolis, MN, USA) in DMEM $1 \mathrm{~g} / \mathrm{L}$ glucose, glutaMAX, and pyruvate on $1 \times 10^{4} \mathrm{hMSCs} / \mathrm{cm}^{2} / 0.5 \mathrm{~mL}$. After 21 days, an extracellular matrix built by the chondrocytes was stained with alcian blue (Sigma Aldrich, Steinheim, Germany). Osteogenic differentiation was achieved using $10 \mathrm{nM}$ dexamethason, $100 \mu \mathrm{M}$ L-ascorbic acid 2-phosphate sesquimagnesic salt, and $10 \mathrm{mM}$ beta-glycerophosphate (Sigma Aldrich, Steinheim, Germany) in DMEM $1 \mathrm{~g} / \mathrm{L}$ glucose, glutaMAX, and pyruvate on $2.5 \times 10^{3} \mathrm{hMSCs} / \mathrm{cm}^{2} / 0.5 \mathrm{~mL}$. After 14 days, calcium deposition from osteoblasts were investigated via light microscopy.

\subsection{Isolation and Differentiation of Human Monocyte-Derived Macrophages (hMDM) and THP-1 Cells}

Human monocytes were isolated from buffy coats of healthy donors. All donors gave written consent, and the isolation was approved by an ethics commission (University of Leipzig no. 427/20-ek). Blood from buffy coats was filtered once with a nylon sieve and CD14+ isolation was performed using the StraightFrom ${ }^{\circledR}$ Buffy Coat CD14 MicroBead Kit (Miltenyi Biotec, Bergisch Gladbach, Germany) according to the manufacturer's instructions. Isolated monocytes were resuspended in RPMI-1640, glutaMAX, and HEPES (Thermo Fisher Scientific, Waltham, MA, USA) plus 10\% FCS and 1\% penicillin/streptomycin and used for experiments immediately after isolation.

For macrophage differentiation, the freshly isolated monocytes were incubated with GM-CSF for 7 days. Here, cells were seeded in suspension flasks at $1 \times 10^{6}$ cells $/ \mathrm{mL}$ in RPMI-1640 medium with $100 \mathrm{ng} / \mathrm{mL}$ GM-CSF. After $72 \mathrm{~h}$, the medium was changed by centrifuging at $450 \times g$ and resuspending the cell pellet in fresh RPMI-1640 medium with $100 \mathrm{ng} / \mathrm{mL}$ GM-CSF, followed by plating the cells at $5 \times 10^{5}$ cells $/ \mathrm{cm}^{2} / 0.5 \mathrm{~mL}$. Cells were incubated for additional $96 \mathrm{~h}$ to gain macrophages (hMDM M0).

THP-1 cells (ATCC ${ }^{\circledR}$ TIB-202 ${ }^{\mathrm{TM}}$ ) were seeded at $5 \times 10^{4}$ cells $/ \mathrm{cm}^{2} / 0.5 \mathrm{~mL}$ in RPMI1640 medium with $100 \mathrm{ng} / \mathrm{mL}$ PMA. After $24 \mathrm{~h}$ incubation, the medium was replaced with fresh RPMI-1640 medium for an additional $24 \mathrm{~h}$ to gain macrophages (THP-1 M0).

\subsection{Polarization of Macrophages to M1 without and with $P G E_{2}$ or M1-Primed hMSC-Conditioned Medium (hMSC CM)}

After differentiation, both cell types (hMDM M0, THP-1 M0) were polarized to M1 macrophages by replacing the culture medium with the DMEM medium containing $20 \mathrm{ng} / \mathrm{mL}$ IFN- $\gamma$ and $100 \mathrm{ng} / \mathrm{mL}$ LPS without or with $\mathrm{PGE}_{2}(5 \mathrm{ng} / \mathrm{mL})$. Alternatively, the M1-primed hMSC-conditioned medium was mixed in equal parts with the DMEM medium containing $40 \mathrm{ng} / \mathrm{mL}$ IFN $-\gamma$ and $200 \mathrm{ng} / \mathrm{mL}$ LPS and added to the M0 macrophages. After $24 \mathrm{~h}$, the supernatant was collected, centrifuged, and stored at $-80^{\circ} \mathrm{C}$ for further analysis of cytokine secretion. The M1 supernatant was also used to trigger hMSCs for the preparation of the M1-primed hMSC-conditioned medium (see Section 2.4). Cells were washed with PBS and lysed for RNA isolation.

\subsection{Preparation of hMSC-Conditioned Medium}

hMSCs were seeded at $2.5 \times 10^{3}$ cells $/ \mathrm{cm}^{2} / 0.5 \mathrm{~mL}$ in an equal mix of the DMEM medium and conditioned medium from polarized THP-1 M1 macrophages (described in 
Section 2.3). hMSCs were incubated for $24 \mathrm{~h}$ and the resulting supernatant was centrifuged and stored at $-80{ }^{\circ} \mathrm{C}$.

\subsection{Testing of the Influence of hMSC on Readily Polarized M1 Macrophages}

Macrophages were polarized to M1 macrophages as described in Section 2.3. Unless stated otherwise, the M1 macrophages were incubated for additional $24 \mathrm{~h}$ in the presence of (a) the conditioned hMSC medium, (b) hMSCs in direct coculture, (c) $5 \mathrm{ng} / \mathrm{mL} \mathrm{PGE}$, or (d) only the DMEM medium as a control by replacing only half of the culture media to maintain the inflammatory environment. Samples were taken as described above.

For the direct coculture, $5 \times 10^{3}$ cells $/ \mathrm{cm}^{2} / 0.5 \mathrm{~mL}$ hMSCs were added directly to the M1 macrophage-containing wells.

\subsection{Analysis of Cytokine Secretion and Gene Expression}

Cytokines in the supernatants from the different experiments were analyzed by quantitative immunoassays (ELISA) for TNFalpha, IL-1ra, IL-10 (R\&D Systems, Inc., Minneapolis, MN, USA), PGE 2 (Cayman Chemical, Ann Arbor, MI, USA), and TSG-6 (RayBiotech, Inc., Peachtree Corners, GA, USA) according to the manufacturers' instructions. Present cytokine concentrations in M1-primed hMSC CM were deducted from the concentrations measured in the polarization experiments to identify cytokine secretion of macrophages upon treatment.

RNA was isolated using the RNeasy Mini Kit (Qiagen, Hilden, Germany). cDNA was synthesized using the GoScript ${ }^{\mathrm{TM}}$ Reverse Transcription System (Promega, Madison, WI, USA) from $1000 \mathrm{ng}$ of total RNA. Quantitative PCR was performed using PowerUp ${ }^{\mathrm{TM}}$ SYBR Green Master Mix (Thermo Fisher Scientific, Waltham, MA, USA) and exon-exon spanning primers for CCL-17, CCL-22, CCR7, IL-1ra, IL-10, RPL37A, and TNFalpha. The sense and antisense primer are as follows: CCL-17, $5^{\prime}$-CAGGGATGCCATCGTTTTTGT$3^{\prime}$, and $5^{\prime}$ - CGGGAGACAGTCAGGAGTCT-3' ${ }^{\prime}$ CCL-22, 5'-GGCGTGGTGTTGCTAACCT- ${ }^{\prime}$, and $5^{\prime}$-GCCACGGTCATCAGAGTAGG-3'; CCR7, 5' ${ }^{\prime}$-TGGTGGTGGCTCTCCTTGTC-3', and $5^{\prime}$-TGTGGTGTTGTCTCCGATGTAATC- ${ }^{\prime}$; IL-1ra, $5^{\prime}$-TCCGCAGTCACCTAATCACTC$3^{\prime}$, and $5^{\prime}$-AACATCCCAGATTCTGAAGGC- $3^{\prime}$; IL-10, 5'-TACGGCGCTGTCATCGATTT- ${ }^{\prime}$, and $5^{\prime}$-TAGAGTCGCCACCCTGATGT- ${ }^{\prime}$; RPL37A, $5^{\prime}$-ATTGAAATCAGCCAGCACGC- ${ }^{\prime}$, and $5^{\prime}$-AGGAACCACAGTGCCAGATCC- $3^{\prime}$; TNFalpha, $5^{\prime}$-TCTTCTCGAACCCCGAGTGAC- $3^{\prime}$, and $5^{\prime}$-GGTACAGGCCCTCTGATGGC- $3^{\prime}$. Gene expression data were evaluated using the $\Delta \Delta$-CT-method [19] using RPL37A as a previously described housekeeper gene for THP-1 cells [20].

\subsection{Statistical Analysis}

Statistical analysis was performed using analysis of variance (ANOVA) with Sidak or Dunnett's post hoc test. Statistical significance was determined as ${ }^{*}=p<0.05 ;{ }^{* *}=p<0.01$; and ${ }^{* * *}=p<0.001$. All experiments were performed in triplicates.

\section{Results}

\subsection{M1-Conditioned Medium Induces $P G E_{2}$ Secretion in hMSCS}

Human MSCs derived from five different bone marrow donations were seeded with conditioned media of THP-1 M1 macrophages (M1-primed) and the resulting M1-primed hMSC-conditioned media (hMSC CM) was collected. The M1-primed hMSC CM and non-treated controls were analyzed for their secretion of PGE 2 , IL-1ra, IL-10, and TSG-6, all described to be potentially secreted by hMSCs upon stimulation and having an impact on macrophages [8,9]. Upon hMSC stimulation with the conditioned medium from THP-1 M1 macrophages (M1 CM), the measured IL-1ra and IL-10 concentrations did not exceed the initial concentrations from the M1 CM, and no TSG-6 secretion was detected in any sample. However, $\mathrm{PGE}_{2}$ secretion was strongly induced in the cells of all five hMSC donations with no significant difference between the donations, whereas no $\mathrm{PGE}_{2}$ was found in the M1 $\mathrm{CM}$ (Figure 1). As only $\mathrm{PGE}_{2}$ was seen to be regulated upon the activation of hMSCs using 
M1 CM, immunomodulation of M1 macrophages via PGE 2 was also included in our study and compared to the effects of M1-primed hMSC CM.
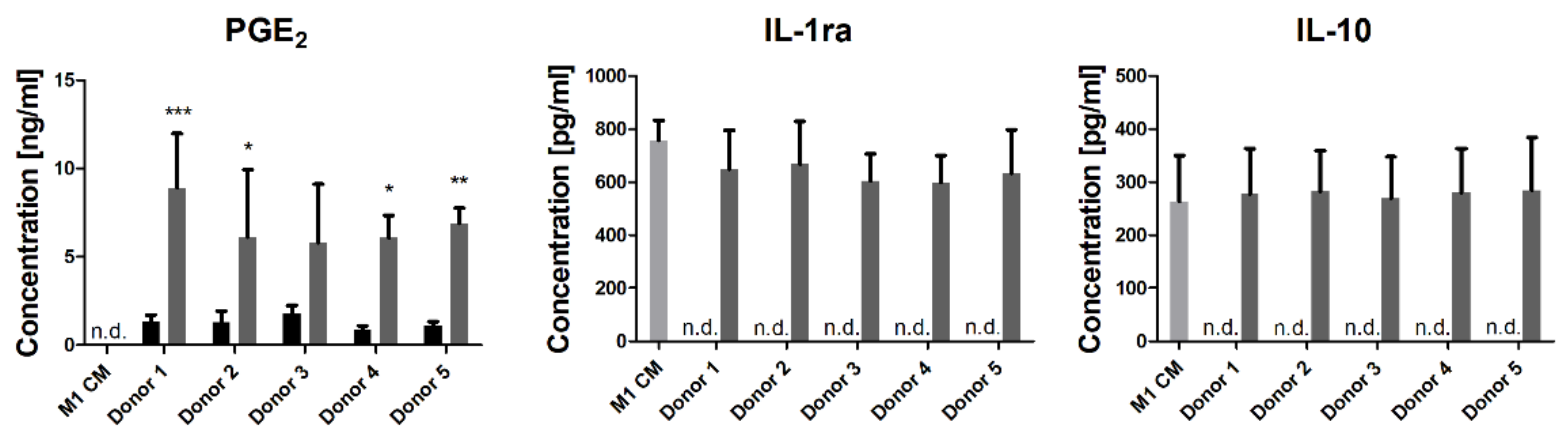

Figure 1. IL-1ra, IL-10, and PGE2 secretion of hMSCs upon stimulation with the conditioned medium of polarized THP-1 M1 macrophages. Present initial concentrations from M1 CM are depicted for reference (light gray bars). hMSCs from five donors were incubated for $24 \mathrm{~h}$ with the M1-conditioned medium (+M1 CM, dark gray bars) or without (black bars). Significance was determined between control and treatment for each donor using two-way ANOVA and Sidak's post hoc test. Between donors, no significant difference was found in any case. TSG-6 was not detectable in all controls and treatments. ${ }^{*}=p<0.05 ;{ }^{* *}=p<0.01$; and ${ }^{* * *}=p<0.001$. $n=3$. Abbreviation: n.d., non-detectable.

\section{2. hMSC Modulate the Cytokine Secretion of Polarizing Macrophages}

THP-1 M0 as well as hMDM M0 macrophages were polarized towards M1 with or without the addition of M1-primed hMSC-conditioned medium or $\mathrm{PGE}_{2}$ (Figure 2). For comparison, a fresh culture medium spiked with $5 \mathrm{ng} / \mathrm{mL} \mathrm{PGE} 2$ was used, as this closely resembles the $\mathrm{PGE}_{2}$ concentration in the treatment with the two-fold diluted hMSCconditioned medium (see Section 2.3). The gene expression analysis of the polarized THP-1 M1 macrophages after $24 \mathrm{~h}$ showed that neither the treatment with hMSC-conditioned medium nor $\mathrm{PGE}_{2}$ led to a significant inhibition of the gene expression of the proinflammatory markers TNFalpha and CCR7. Instead, CCR7 expression was significantly increased by $\mathrm{PGE}_{2}$ (Figure 2A). The gene expression of the anti-inflammatory markers IL-1ra, IL-10, and CCL-17 was not affected by the conditioned medium or PGE 2 . Only CCL-22 was significantly upregulated upon both treatments compared to the untreated control. Interestingly, the secretion of the inflammatory cytokine TNFalpha was strongly inhibited when THP-1 macrophages were polarized under the influence of the hMSC-conditioned medium as well as by $\mathrm{PGE}_{2}$ alone (Figure 2B). This effect was confirmed on hMDMs using the same stimulatory conditions (Figure 2C). At the same time, a slight but significant increase of the secretion of the anti-inflammatory marker IL-1ra can be seen in both cell types, but the effects of the treatment with $\mathrm{PGE}_{2}$ and the more complex conditioned medium differ (Figure 2B,C). Additionally, IL-10 was significantly elevated in THP-1 cells by both M1-primed hMSC CM and $\mathrm{PGE}_{2}$, but not in hMDMs, hinting at a difference between the cell line and primary cells.

\subsection{M1-Primed hMSCs Fail to Modulate the Cytokine Secretion of Polarized Macrophages}

Macrophages were polarized towards M1 using LPS and IFN- $\gamma$, as described in Section 2.3. After polarization for $24 \mathrm{~h}, 50 \%$ of the medium was replaced with control medium, spiked medium with $5 \mathrm{ng} / \mathrm{mL} \mathrm{PGE}_{2}$, or conditioned hMSC medium to maintain the inflammatory environment, and cells were incubated for another $24 \mathrm{~h}$.

The gene expression of treated THP-1 M1 macrophages was compared to the control. Both the $\mathrm{PGE}_{2}$ and hMSC-conditioned medium appeared to significantly upregulate the gene expression of CCR7, while the expression of TNFalpha remained unaffected (Figure 3A). The expressions of the anti-inflammatory marker genes CCL-17 and IL-1ra were significantly induced by both treatments. CCL-22 was also significantly induced by $\mathrm{PGE}_{2}$ and appeared less elevated upon treatment with the hMSC-conditioned medium. Interestingly, IL-10 gene expression was unaffected by treatment with $\mathrm{PGE}_{2}$ and the hMSCconditioned medium. The secretion levels of TNFalpha and IL-10 appeared unaffected 
in THP-1 M1 macrophages as well as in hMDMs. However, a significantly elevated IL1ra secretion could be detected in hMDMs under the influence of $\mathrm{PGE}_{2}$ suggesting that M1-primed hMSC CM dampens the effect of $\mathrm{PGE}_{2}$ (Figure 3B,C).

A
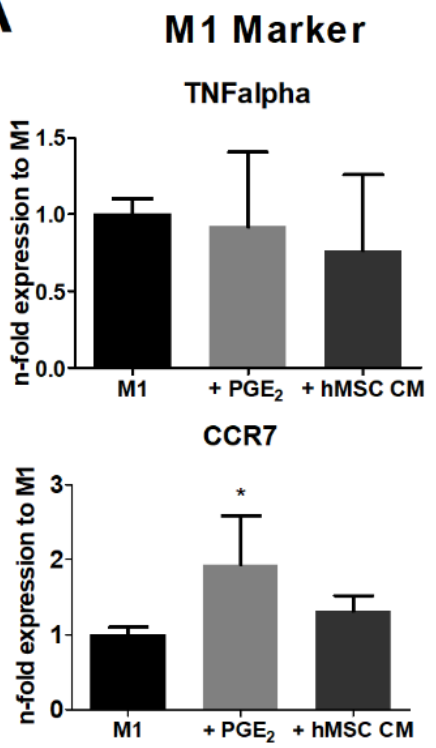

B

M1 Marker

TNFalpha

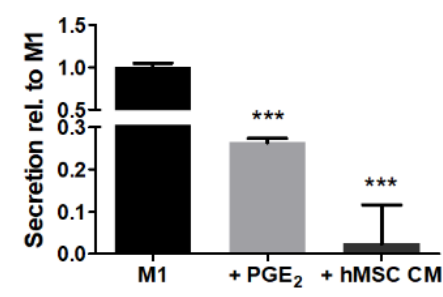

C

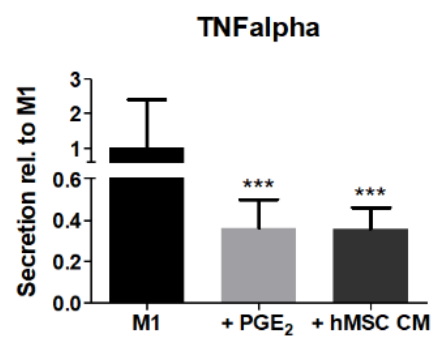

M1 Marker

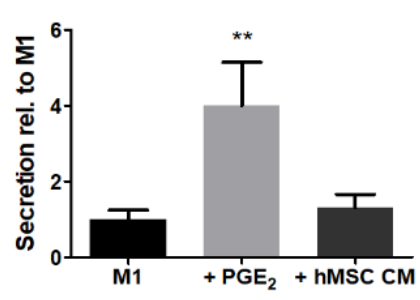

M2 Marker

IL-1ra
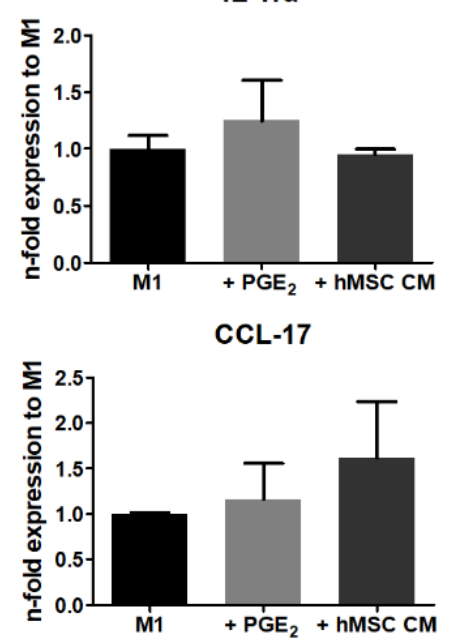

IL-10
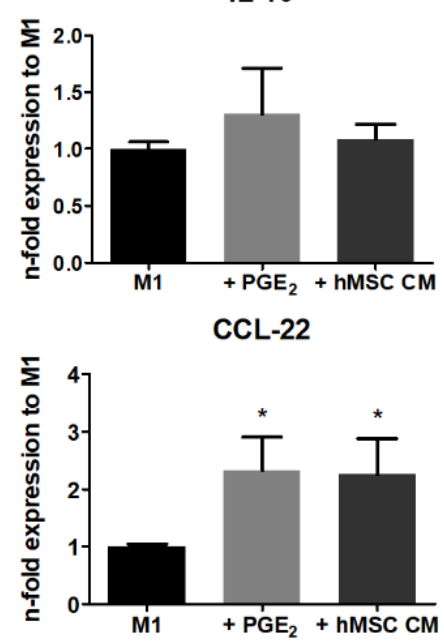

\section{M2 Marker}

IL-1 ra

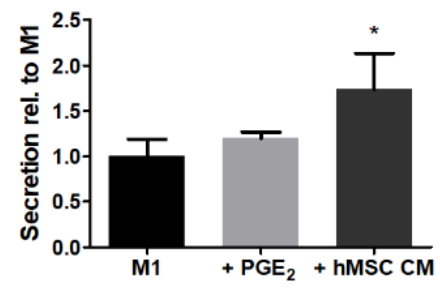

IL-10

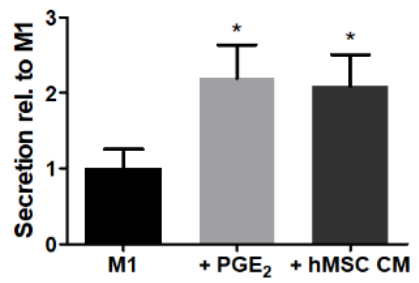

M2 Marker

Figure 2. $\mathrm{PGE}_{2}$ and conditioned medium of hMSCs modulate the M1 polarization of macrophages. Macrophages were polarized to M1 without (M1, black bars) or with $5 \mathrm{ng} / \mathrm{mL} \mathrm{PGE}_{2}\left(+\mathrm{PGE}_{2}\right.$, light grey bars) or the conditioned medium of M1-primed hMSCs (+hMSC CM, dark grey bars). (A) THP-1 gene expression upon M1 polarization after $24 \mathrm{~h}$. Displayed are proinflammatory genes, TNFalpha and CCR7, and anti-inflammatory genes, CCL-17, CCL-22, IL-1ra, and IL-10. (B) Cytokine secretion of THP-1 macrophages upon M1 polarization after $24 \mathrm{~h}$. Displayed are the proinflammatory cytokines, TNFalpha, and the anti-inflammatory cytokines IL-1ra and IL-10. (C) Cytokine secretion of primary macrophages (hMDMs) upon M1 polarization after $24 \mathrm{~h}$. Statistical significance was determined against M1 using one-way ANOVA and Dunnett's post hoc test. ${ }^{*}=p<0.05 ;{ }^{* *}=p<0.01$; and ${ }^{* *}=p<0.001 . n=3$. 
A
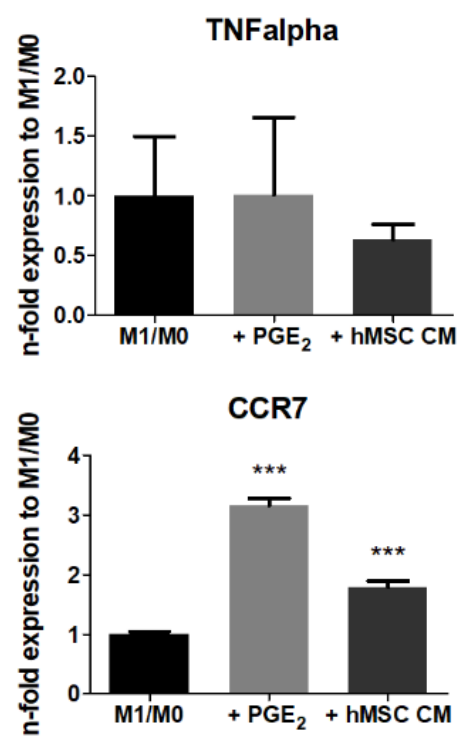

B
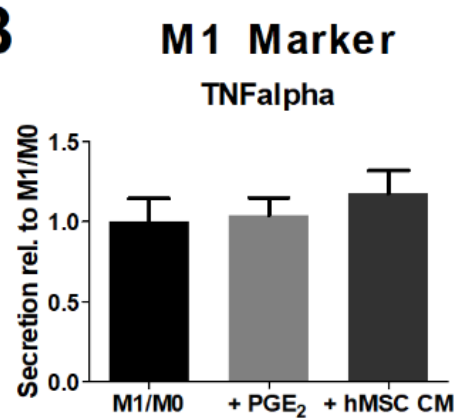

C

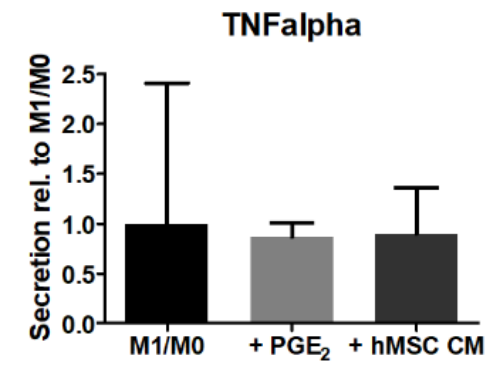

M2 Marker
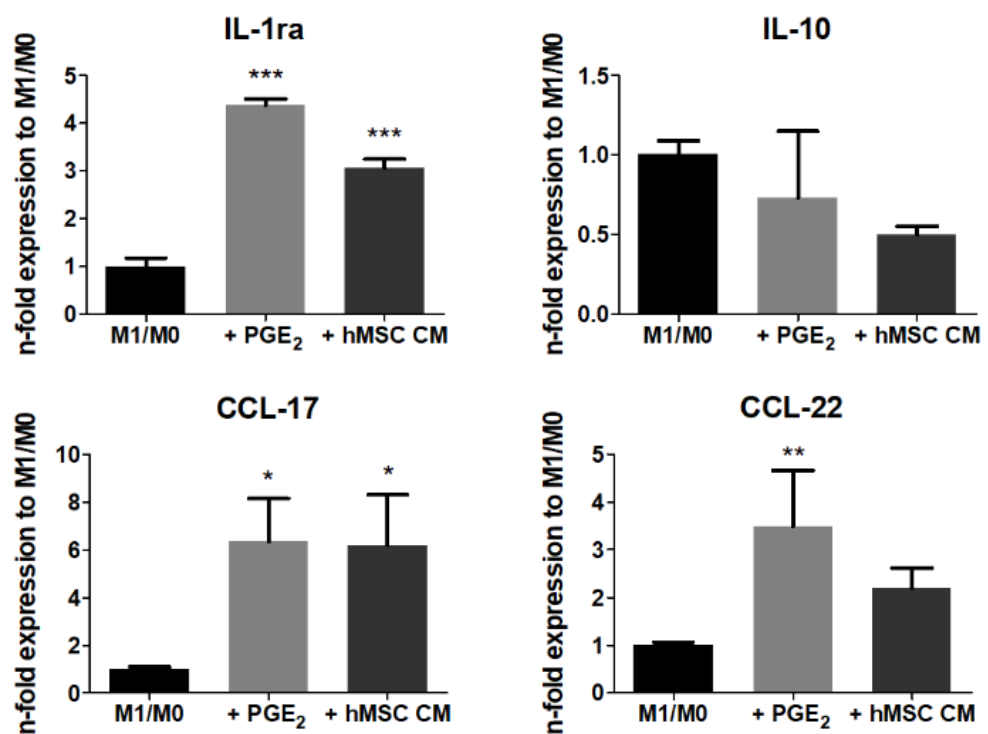

M2 Marker

IL-1ra

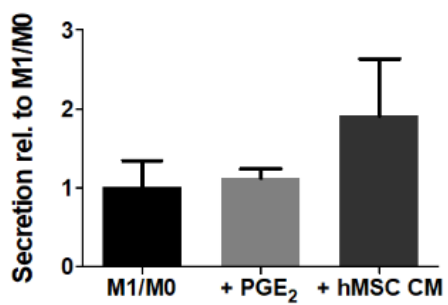

IL-10

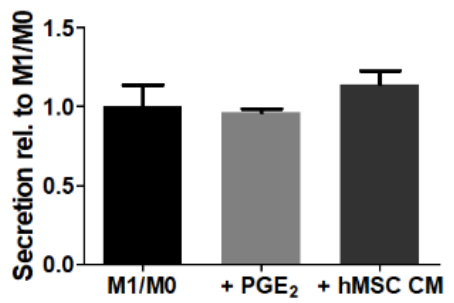

\section{M2 Marker}

IL-1ra

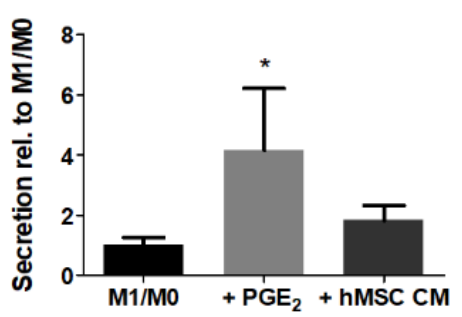

IL-10

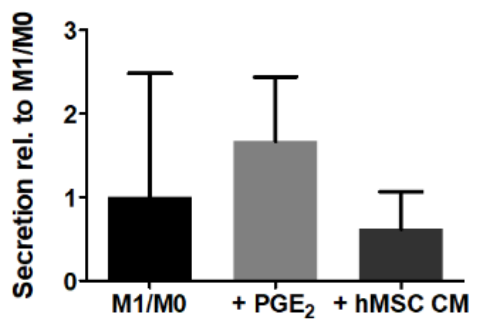

Figure 3. hMSCs modulate the gene expression of M1-polarized macrophages but fail to modulate cytokine secretion. Polarized M1 macrophages were incubated with the control medium (M1/M0, black bars), the spiked medium containing $5 \mathrm{ng} / \mathrm{mL} \mathrm{PGE}_{2}\left(+\mathrm{PGE}_{2}\right)$, light gray bars), or the conditioned medium of M1-primed hMSCs (+hMSC CM, dark grey bars) for $24 \mathrm{~h}$ and compared to the control. (A) THP-1 gene expression fold increase upon incubation without or with PGE 2 or hMSC CM. Displayed are proinflammatory genes, TNFalpha and CCR7, and anti-inflammatory genes, CCL-17, CCL-22, IL-1ra, and IL-10. (B) Cytokine secretion of THP-1 macrophages upon incubation of polarized M1 macrophages without or with $\mathrm{PGE}_{2}$ or hMSC CM. Displayed are proinflammatory cytokine, TNFalpha, and anti-inflammatory cytokines, IL-1ra and IL-10. (C) Cytokine secretion of primary macrophages (hMDMs) upon incubation of polarized M1 macrophages without or with $\mathrm{PGE}_{2}$ or hMSC CM. Statistical significance was determined against the control using one-way ANOVA and Dunnett's post hoc test. ${ }^{*}=p<0.05 ;{ }^{* *}=p<0.01$; and ${ }^{* * *}=p<0.001 . n=3$. 
In an additional experiment, hMSCs were directly cocultured with polarized THP-1 M1 macrophages and effects on gene expression and cytokine secretion were compared to M1 macrophages treated with the hMSC-conditioned medium. In regard to the investigated genes and cytokines, no additional effects were observed in the coculture (Supplementary Materials Figure S2).

\section{Discussion}

Upon stimulation with THP 1 M1-conditioned media, in hMSCs of all five donations, only the secretion of $\mathrm{PGE}_{2}$ was induced. Indeed, $\mathrm{PGE}_{2}$ was particularly found to indicate therapeutic efficacy of human bone marrow MSCs in a traumatic brain injury study [21]. Maggini et al. described a pathway of TNFalpha regulating $\mathrm{PGE}_{2}$ via the binding to the specific receptor TNFR- 1 and subsequent induction of NF-kB and cyclooxygenase-2 (COX-2) expression in MSCs [15]. Interestingly, IL-10, IL-1ra, and TSG6 secretion by hMSCs was not detectable. Previous studies showed that MSCs secrete all three factors in inflammatory scenarios including upon stimulation with macrophage-conditioned media [22-24]. However, high degrees of variability need to be considered between donor species and treatment protocols. In addition, variations between human donations and even within tissue heterogeneity of MSCs and their secretion profile have been documented [23,25]. Hence, a sampling bias cannot be ruled out.

The influence of hMSCs on macrophage polarization was investigated in two different scenarios. First, the hMSC-conditioned medium was added to the M1 macrophage polarization, which resulted in changes in the inflammatory secretion profile. Secreted factors of stimulated hMSC modulate the M1 polarization of macrophages by inhibiting the secretion of TNFalpha-a prominent proinflammatory cytokine secreted by M1 macrophages. Additionally, an induction of typical M2 phenotype-related markers can be seen. Attenuation of TNFalpha secretion was previously observed in a coculture of human MSCs and THP-1 macrophages as well as human MDMs during M1 polarization [17]. In alignment with our findings, Gray et al. reported that human MSCs stimulated with inflammatory triggers inhibit TNFalpha secretion in human MDMs during M1 polarization [11,26]. Wall et al. hypothesized a pathway in which $\mathrm{PGE}_{2}$ inhibits LPS-mediated TNFalpha expression while

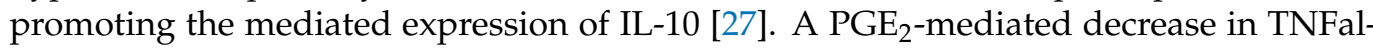
pha secretion and increase in IL-10 and IL-1ra via EP4 was described in polarized mouse macrophages [28]. Elevated CCR7 expression via $\mathrm{PGE}_{2}$ in macrophages was previously described by Tanné et al. in Mono-MAC-1 cells [29]. The receptor CCR7 has been described to facilitate migration of immune cells towards the lymphatic system through its ligands [30]. Effects on macrophage expression of CCL-17 and CCL-22 are less covered in the literature. Interestingly, in monocyte-derived dendritic cells, $\mathrm{PGE}_{2}$ was shown to upregulate both CCL-17 and CCL-22, which in turn attract T helper 2 (Th2) cells [31]. Th2 cells produce anti-inflammatory cytokines, such as IL-4 and IL-13, thus mediating inflammation. This suggests not only that proinflammatory cytokine secretion of M1 polarizing macrophages is inhibited by $\mathrm{PGE}_{2}$ or hMSCs, but also that the haptotaxis of these macrophages away from the inflammatory site and the attraction of Th2 cells that promote M2 polarization of unpolarized macrophages are encouraged. Interestingly, Merimi et al. showed that inflammatory cytokine licensing of human adipose tissue-derived MSCs above passage 2 yielded less expression of immunomodulatory genes IDO1 and PGE2-corresponding PTGS2, suggesting that differently aged MSCs have different roles in wound healing [7]. Regarding the secretome, our study did not find noticeable variations between passages. A broadband secretome analysis of MSC donations regarding the passages can shed further light on these differences. By comparing the results of the hMSC-conditioned medium with that of $\mathrm{PGE}_{2}$, we conclude that hMSCs modulate the M1 polarization mainly via $\mathrm{PGE}_{2}$, supporting previous studies $[16,18]$. Nevertheless, other soluble factors might be involved. Since factors indicated by other researchers, like TSG-6, were not secreted by hMSCs in our experiments, further in-depth studies are needed. 
In the second scenario, the influence of hMSCs on polarized M1 macrophages was investigated in which no significant changes in the selected secretion profile were observed, yet significant changes in the gene expression profiles were found. In contrast to our results, Manferdini et al. showed that human MSCs reduced TNFalpha, IL-1B, and IL-6 secretion in polarized human MDM M1 macrophages, which strongly correlated with the treatment with $\mathrm{PGE}_{2}$ alone [16]. Another study concluded via the mRNA expression ratio of inducible nitric oxide synthase (iNOS) and arginase I that conditioned media of MSCs stimulated murine macrophages from an M1 to an M2 subtype in vitro [28]. However, it is noteworthy that in both studies M1 macrophages were polarized with IFN- $\gamma$ only. However, Guillén et al. reported that conditioned media from unprimed human adipose tissue-derived MSCs regulated human MDM M1-polarized macrophages by inhibiting TNFalpha secretion and increasing IL-10 and TGF-B secretion when polarizing macrophages with IFN- $\gamma$ and LPS [32]. This contrasting finding may be the result of varying immunomodulatory capacities of MSCs from different sources. In comparison to the results obtained with $\mathrm{PGE}_{2}$ and the hMSC-conditioned medium when added during the polarization of macrophages (Section 3.2), the treatment of already polarized macrophages with $\mathrm{PGE}_{2}$ or the hMSCconditioned medium led to an elevated gene expression of anti-inflammatory marker genes but failed to induce the strong inhibitory effect on the TNFalpha secretion of THP-1 M1 macrophages as well as on primary hMDMs.

In coculture experiments, no additional effects on gene expression and cytokine secretion could be observed compared to the treatment with the conditioned hMSC medium. This is in line with other studies that concluded that hMSCs exert their immunomodulating effects mainly via soluble factors $[3,33]$.

\section{Conclusions}

Our results of the direct comparison of human bone marrow MSC effects on polarizing vs. polarized inflammatory macrophages demonstrated that both scenarios are influenced by immunologically activated hMSCs, but in a different way. The results show that hMSC have no effect on the protein secretion profile of already polarized proinflammatory macrophages but inhibited the proinflammatory polarization of naive macrophages as seen by the strong inhibition of the TNFalpha secretion.

The direct comparison of the two different scenarios under the same culture conditions in vitro strongly demonstrated the significance of a clear in vitro study design and statement. In addition, our results show that the use of the THP- 1 cell line is adequate for the in vitro testing of immunomodulating effects of hMSCs, as the results using primary hMDMs are comparable.

Following our conclusion that MSCs can only modulate the inflammatory environment in vivo by inhibiting the proinflammatory polarization of naive macrophages and cannot influence already polarized proinflammatory macrophages, we can refine the therapeutic knowledge of hMSCs in inflammatory diseases. Nevertheless, more effort on clarifying the role of distinct soluble factors secreted by inflammatory primed MSCs in orchestrating the shift in the inflammatory environment is needed. In addition, more studies are needed to compare the immunomodulatory capacity of hMSCs of different origin. We agree with the ISCT to add markers to the characterization panel of MSCs, which clearly define their immunomodulating potential as part of their routine characterization. Our approach of stimulating hMSCs in vitro with the cell secretome of a stable macrophage cell line (THP-1) to activate the hMSCs for their immunomodulatory factor secretion would allow for comparable studies between laboratories to develop assays for the routine testing of the immunomodulating capacity of hMSCs.

Studies using more than the expression and secretion markers used in our experiments may shed further light on the influence of hMSC on macrophages in different stages and may further help in elucidating the role of hMSC therapies in inflammatory diseases and the potential development of drugs based on hMSC effects on macrophages. 
Supplementary Materials: The following are available online at https://www.mdpi.com/article/10 .3390/immuno1040036/s1; Figure S1: Light microscopic images of adipogenic, chondrogenic, and osteogenic differentiation of hMSCs including non-treated controls. Figure S2: Gene expression and cytokine secretion of a coculture with hMSCs and M1 polarized THP-1 macrophages in comparison to M1 modulation with hMSC conditioned media.

Author Contributions: Conceptualization, C.I., S.K. and S.S.; methodology, C.I., S.K. and B.A.; validation, S.S.; investigation, C.I.; writing-original draft preparation, C.I.; writing-review and editing, S.K., S.S. and J.S.; project administration, J.S.; funding acquisition, J.S. All authors have read and agreed to the published version of the manuscript.

Funding: This work was funded by the Fraunhofer ATTRACT project PrimBioCer, grant number 068-692000.

Institutional Review Board Statement: The study was conducted according to the guidelines of the Declaration of Helsinki, and the study was approved by the ethics commission of the University of Leipzig (no. 486/17-ek-001 and 427/20-ek).

Informed Consent Statement: All donors gave written consent to the usage of resulting waste material of their bone marrow and whole blood donations for the research purposes. Waste material of donations was acquired without receiving personal data of the donors.

Data Availability Statement: The data presented in this study are available on request from the corresponding author.

Acknowledgments: We thank Lysann Reichelt for the technical support and the Institute of Transfusion Medicine in Leipzig for the fruitful scientific discussions.

Conflicts of Interest: The authors declare no conflict of interest. The funders had no role in the design of the study; in the collection, analyses, or interpretation of data; in the writing of the manuscript; or in the decision to publish the results.

\section{References}

1. Shapouri-Moghaddam, A.; Mohammadian, S.; Vazini, H.; Taghadosi, M.; Esmaeili, S.-A.; Mardani, F.; Seifi, B.; Mohammadi, A.; Afshari, J.T.; Sahebkar, A. Macrophage plasticity, polarization, and function in health and disease. J. Cell. Physiol. 2018, 233, 6425-6440. [CrossRef] [PubMed]

2. Prockop, D.J. Concise review: Two negative feedback loops place mesenchymal stem/stromal cells at the center of early regulators of inflammation. Stem Cells 2013, 31, 2042-2046. [CrossRef] [PubMed]

3. Pajarinen, J.; Lin, T.; Gibon, E.; Kohno, Y.; Maruyama, M.; Nathan, K.; Lu, L.; Yao, Z.; Goodman, S.B. Mesenchymal stem cell-macrophage crosstalk and bone healing. Biomaterials 2019, 196, 80-89. [CrossRef] [PubMed]

4. Bernardo, M.E.; Fibbe, W.E. Mesenchymal stromal cells: Sensors and switchers of inflammation. Cell Stem Cell 2013, 13, $392-402$. [CrossRef] [PubMed]

5. Le Blanc, K.; Davies, L.C. Mesenchymal stromal cells and the innate immune response. Immunol. Lett. 2015, 168, 140-146. [CrossRef] [PubMed]

6. Kabat, M.; Bobkov, I.; Kumar, S.; Grumet, M. Trends in mesenchymal stem cell clinical trials 2004-2018: Is efficacy optimal in a narrow dose range? Stem Cells Transl. Med. 2020, 9, 17-27. [CrossRef] [PubMed]

7. Merimi, M.; Buyl, K.; Daassi, D.; Rodrigues, R.M.; Melki, R.; Lewalle, P.; Vanhaecke, T.; Fahmi, H.; Rogiers, V.; Lagneaux, L.; et al. Transcriptional Profile of Cytokines, Regulatory Mediators and TLR in Mesenchymal Stromal Cells after Inflammatory Signaling and Cell-Passaging. Int. J. Mol. Sci. 2021, 22, 7309. [CrossRef]

8. Li, X.; Yue, S.; Luo, Z. Mesenchymal stem cells in idiopathic pulmonary fibrosis. Oncotarget 2017, 8, 102600-102616. [CrossRef]

9. Prockop, D.J.; Oh, J.Y. Mesenchymal stem/stromal cells (MSCs): Role as guardians of inflammation. Mol. Ther. J. Am. Soc. Gene Ther. 2012, 20, 14-20. [CrossRef]

10. Ren, G.; Su, J.; Zhang, L.; Zhao, X.; Ling, W.; L'huillie, A.; Zhang, J.; Lu, Y.; Roberts, A.I.; Ji, W.; et al. Species variation in the mechanisms of mesenchymal stem cell-mediated immunosuppression. Stem Cells 2009, 27, 1954-1962. [CrossRef]

11. Gray, A.; Schloss, R.S.; Yarmush, M. Donor variability among anti-inflammatory pre-activated mesenchymal stromal cells. Technology 2016, 4, 201-215. [CrossRef] [PubMed]

12. Mennan, C.; Garcia, J.; Roberts, S.; Hulme, C.; Wright, K. A comprehensive characterisation of large-scale expanded human bone marrow and umbilical cord mesenchymal stem cells. Stem Cell Res. Ther. 2019, 10, 99. [CrossRef] [PubMed]

13. Siegel, G.; Kluba, T.; Hermanutz-Klein, U.; Bieback, K.; Northoff, H.; Schäfer, R. Phenotype, donor age and gender affect function of human bone marrow-derived mesenchymal stromal cells. BMC Med. 2013, 11, 146. [CrossRef] 
14. Asami, T.; Ishii, M.; Fujii, H.; Namkoong, H.; Tasaka, S.; Matsushita, K.; Ishii, K.; Yagi, K.; Fujiwara, H.; Funatsu, Y.; et al. Modulation of murine macrophage TLR7/8-mediated cytokine expression by mesenchymal stem cell-conditioned medium. Mediat. Inflamm. 2013, 2013, 264260. [CrossRef] [PubMed]

15. Maggini, J.; Mirkin, G.; Bognanni, I.; Holmberg, J.; Piazzón, I.M.; Nepomnaschy, I.; Costa, H.; Cañones, C.; Raiden, S.; Vermeulen, M.; et al. Mouse bone marrow-derived mesenchymal stromal cells turn activated macrophages into a regulatory-like profile. PLOS ONE 2010, 5, e9252. [CrossRef] [PubMed]

16. Manferdini, C.; Paolella, F.; Gabusi, E.; Gambari, L.; Piacentini, A.; Filardo, G.; Fleury-Cappellesso, S.; Barbero, A.; Murphy, M.; Lisignoli, G. Adipose stromal cells mediated switching of the pro-inflammatory profile of M1-like macrophages is facilitated by PGE2: In vitro evaluation. Osteoarthr. Cartil. 2017, 25, 1161-1171. [CrossRef]

17. Tedesco, S.; de Majo, F.; Kim, J.; Trenti, A.; Trevisi, L.; Fadini, G.P.; Bolego, C.; Zandstra, P.W.; Cignarella, A.; Vitiello, L. Convenience versus Biological Significance: Are PMA-Differentiated THP-1 Cells a Reliable Substitute for Blood-Derived Macrophages When Studying in Vitro Polarization? Front. Pharmacol. 2018, 9, 71. [CrossRef]

18. Vasandan, A.B.; Jahnavi, S.; Shashank, C.; Prasad, P.; Kumar, A.; Prasanna, S.J. Human Mesenchymal stem cells program macrophage plasticity by altering their metabolic status via a PGE2-dependent mechanism. Sci. Rep. 2016, 6, 38308. [CrossRef] [PubMed]

19. Pfaffl, M.W. A new mathematical model for relative quantification in real-time RT-PCR. Nucleic Acids Res. 2001, $29,2002-2007$. [CrossRef] [PubMed]

20. Maess, M.B.; Sendelbach, S.; Lorkowski, S. Selection of reliable reference genes during THP-1 monocyte differentiation into macrophages. BMC Mol. Biol. 2010, 11, 90. [CrossRef] [PubMed]

21. Kota, D.J.; Prabhakara, K.S.; Toledano-Furman, N.; Bhattarai, D.; Chen, Q.; DiCarlo, B.; Smith, P.; Triolo, F.; Wenzel, P.L.; Cox, C.S.; et al. Prostaglandin E2 Indicates Therapeutic Efficacy of Mesenchymal Stem Cells in Experimental Traumatic Brain Injury. Stem Cells 2017, 35, 1416-1430. [CrossRef]

22. Saldaña, L.; Bensiamar, F.; Vallés, G.; Mancebo, F.J.; García-Rey, E.; Vilaboa, N. Immunoregulatory potential of mesenchymal stem cells following activation by macrophage-derived soluble factors. Stem Cell Res. Ther. 2019, 10, 58. [CrossRef] [PubMed]

23. Ortiz, L.A.; DuTreil, M.; Fattman, C.; Pandey, A.C.; Torres, G.; Go, K.; Phinney, D.G. Interleukin 1 receptor antagonist mediates the antiinflammatory and antifibrotic effect of mesenchymal stem cells during lung injury. Proc. Natl. Acad. Sci. USA 2007, 104, 11002-11007. [CrossRef]

24. Choi, H.; Lee, R.H.; Bazhanov, N.; Oh, J.Y.; Prockop, D.J. Anti-inflammatory protein TSG-6 secreted by activated MSCs attenuates zymosan-induced mouse peritonitis by decreasing TLR2/NF-KB signaling in resident macrophages. Blood 2011, 118, 330-338 [CrossRef] [PubMed]

25. Phinney, D.G.; Kopen, G.; Righter, W.; Webster, S.; Tremain, N.; Prockop, D.J. Donor variation in the growth properties and osteogenic potential of human marrow stromal cells. J. Cell. Biochem. 1999, 75, 424-436. [CrossRef]

26. Gray, A.; Maguire, T.; Schloss, R.; Yarmush, M.L. Identification of IL-1beta and LPS as optimal activators of monolayer and alginate-encapsulated mesenchymal stromal cell immunomodulation using design of experiments and statistical methods. Biotechnol. Prog. 2015, 31, 1058-1070. [CrossRef] [PubMed]

27. Wall, E.A.; Zavzavadjian, J.R.; Chang, M.S.; Randhawa, B.; Zhu, X.; Hsueh, R.C.; Liu, J.; Driver, A.; Bao, X.R.; Sternweis, P.C.; et al. Suppression of LPS-Induced TNF-alpha Production in Macrophages by cAMP Is Mediated by PKA-AKAP95-p105. Immunology 2009,2, ra28.

28. Zullo, J.A.; Nadel, E.P.; Rabadi, M.M.; Baskind, M.J.; Rajdev, M.A.; Demaree, C.M.; Vasko, R.; Chugh, S.S.; Lamba, R.; Goligorsky, M.S.; et al. The Secretome of Hydrogel-Coembedded Endothelial Progenitor Cells and Mesenchymal Stem Cells Instructs Macrophage Polarization in Endotoxemia. Stem Cells Transl. Med. 2015, 4, 852-861. [CrossRef]

29. Tanné, B.; Bernier, S.; Dumais, N. CCR7 Receptor Expression in Mono-MAC-1 Cells: Modulation by Liver X Receptor $\alpha$ Activation and Prostaglandin $\mathrm{E}_{2}$. Int. J. Inflamm. 2015, 2015, 201571. [CrossRef]

30. Purvanov, V.; Matti, C.; Samson, G.P.B.; Kindinger, I.; Legler, D.F. Fluorescently Tagged CCL19 and CCL21 to Monitor CCR7 and ACKR4 Functions. Int. J. Mol. Sci. 2018, 19, 3876. [CrossRef]

31. McIlroy, A.; Caron, G.; Blanchard, S.; Frémaux, I.; Duluc, D.; Delneste, Y.; Chevailler, A.; Jeannin, P. Histamine and prostaglandin E up-regulate the production of Th2-attracting chemokines (CCL17 and CCL22) and down-regulate IFN-gamma-induced CXCL10 production by immature human dendritic cells. Immunology 2006, 117, 507-516. [CrossRef] [PubMed]

32. Guillén, M.I.; Platas, J.; Pérez Del Caz, M.D.; Mirabet, V.; Alcaraz, M.J. Paracrine Anti-inflammatory Effects of Adipose TissueDerived Mesenchymal Stem Cells in Human Monocytes. Front. Physiol. 2018, 9, 661. [CrossRef] [PubMed]

33. Hidalgo-Garcia, L.; Galvez, J.; Rodriguez-Cabezas, M.E.; Anderson, P.O. Can a Conversation Between Mesenchymal Stromal Cells and Macrophages Solve the Crisis in the Inflamed Intestine? Front. Pharmacol. 2018, 9, 179. [CrossRef] [PubMed] 